\title{
Consensus report from the 8th International Forum for Liver Magnetic Resonance Imaging
}

\author{
Christoph J. Zech ${ }^{1}$ - Ahmed Ba-Ssalamah ${ }^{2} \cdot$ Thomas Berg $^{3} \cdot$ Hersh Chandarana ${ }^{4,5} \cdot$ Gar-Yang Chau $^{6} \cdot$ Luigi Grazioli $^{7}$. \\ Myeong-Jin Kim ${ }^{8}$. Jeong Min Lee ${ }^{9}$. Elmar M. Merkle ${ }^{1} \cdot$ Takamichi Murakami $^{10}$. Jens Ricke ${ }^{11} \cdot$ Claude B. Sirlin $^{12}$. \\ Bin Song ${ }^{13} \cdot$ Bachir Taouli $^{14} \cdot$ Kengo Yoshimitsu ${ }^{15} \cdot$ Dow-Mu Koh $^{16}$
}

Received: 11 April 2019 / Revised: 27 June 2019 / Accepted: 12 July 2019/Published online: 5 August 2019

(C) The Author(s) 2019

\begin{abstract}
Objectives The 8th International Forum for Liver Magnetic Resonance Imaging (MRI), held in Basel, Switzerland, in October 2017, brought together clinical and academic radiologists from around the world to discuss developments in and reach consensus on key issues in the field of gadoxetic acid-enhanced liver MRI since the previous Forum held in 2013.

Methods Two main themes in liver MRI were considered in detail at the Forum: the use of gadoxetic acid for contrast-enhanced MRI in patients with liver cirrhosis and the technical performance of gadoxetic acid-enhanced liver MRI, both opportunities and challenges. This article summarises the expert presentations and the delegate voting on consensus statements discussed at the Forum. Results and conclusions It was concluded that gadoxetic acid-enhanced MRI has higher sensitivity for the diagnosis of hepatocellular carcinoma (HCC), when compared with multidetector $\mathrm{CT}$, by utilising features of hyperenhancement in the arterial phase and hypointensity in the hepatobiliary phase (HBP). Recent HCC management guidelines recognise an increasing role for gadoxetic acid-enhanced MRI in early diagnosis and monitoring post-resection. Additional research is needed to define the role of HBP in predicting microvascular invasion, to better define washout during the transitional phase in gadoxetic acid-enhanced MRI for HCC diagnosis, and to reduce the artefacts encountered in the arterial phase. Technical developments are being directed to shortening the MRI protocol for reducing time and patient discomfort and toward utilising faster imaging and non-Cartesian free-breathing approaches that have the potential to improve multiphasic dynamic imaging.

Key Points

- Gadoxetic acid-enhanced MRI provides higher diagnostic sensitivity than CT for diagnosing HCC.

- Gadoxetic acid-enhanced MRI has roles in early-HCC diagnosis and monitoring post-resection response.

- Faster imaging and free-breathing approaches have potential to improve multiphasic dynamic imaging.
\end{abstract}

Keywords Gadoxetic acid · Hepatocellular carcinoma $\cdot$ Magnetic resonance imaging

Abbreviations

AASLD

AP

American Association for the

Study of Liver Diseases

Arterial phase

Electronic supplementary material The online version of this article (https://doi.org/10.1007/s00330-019-06369-4) contains supplementary material, which is available to authorized users.

Christoph J. Zech

Christoph.Zech@usb.ch

Takamichi Murakami murataka@med.kobe-u.ac.jp

Extended author information available on the last page of the article
APASL

APHE

BCLC

BW

CT

DN

DP

DWI

EASL-EORTC

ECCM

EOB
Asian Pacific Association for the

Study of the Liver

Arterial phase hyperenhancement

Barcelona Clinic Liver Cancer

Body weight

Computed tomography

Dysplastic nodule

Delayed phase

Diffusion-weighted imaging

European Association for Study

of Liver-European Organisation

for Research and Treatment of Cancer

Extracellular contrast media

Gadoxetic acid 


$\begin{array}{ll}\text { GD } & \text { Gadolinium } \\ \text { Gd-EOB-DTPA } & \text { Gadoxetic acid } \\ \text { HBP } & \text { Hepatobiliary phase } \\ \text { HCC } & \text { Hepatocellular carcinoma } \\ \text { HGDN } & \text { High-grade dysplastic nodule } \\ \text { KLCSG-NCC } & \text { Korean Liver Cancer Study Group } \\ \text { LCSGJ } & \text { Liver Cancer Study Group of Japan } \\ \text { LI-RADS } & \text { Liver Imaging Reporting } \\ & \text { and Data System } \\ \text { MRI } & \text { Magnetic resonance imaging } \\ \text { PVP } & \text { Portal venous phase } \\ \text { RFA } & \text { Radiofrequency ablation } \\ \text { T2W } & \text { T2-weighted } \\ \text { TP } & \text { Transitional phase } \\ \text { TRICKS } & \text { Time-resolved imaging of } \\ & \text { contrast kinetics } \\ \text { TSM } & \text { Transient severe motion } \\ \text { TWIST } & \text { Time-resolved angiography } \\ & \text { with stochastic trajectories } \\ \text { US } & \text { Ultrasound } \\ \text { WO } & \text { Washout }\end{array}$

\section{Introduction}

The 8th International Forum for Liver Magnetic Resonance Imaging (MRI) was held in October 2017 in Basel, Switzerland, and attended by 119 radiologists from Asia $(n=46)$, Australia $(n=4)$, Europe $(n=48)$, North America $(n=20)$, and South America $(n=1)$. Delegates were invited to attend who had knowledge of MRI of the liver, including the use of gadoxetic acid. Two main themes were explored at the Forum: (1) the applications of gadoxetic acid (Primovist@, Eovist $\left.{ }^{\circledR}\right)$ for contrast-enhanced MRI in patients with liver cirrhosis and (2) the technical performance of gadoxetic acid-enhanced liver MRI, including opportunities and challenges. As in previous Forums [1-7], consensus statements on selected topics were proposed based on the available evidence and the expert opinions of participants. The final consensus statements and the voting by delegates are presented in this article. The online supplement includes additional information on delegates' views on current practice in liver MRI, based on a questionnaire that was circulated pre-meeting.

\section{Use of gadoxetic acid in patients with liver cirrhosis}

\section{Washout in the transitional phase}

The 2017-dated Liver Imaging Reporting and Data System (LI-RADS), updated in 2018 [8, 9], defines washout appearance on contrast-enhanced MRI as a visually assessed temporal reduction in the enhancement in tumour relative to the composite liver tissue, resulting in extracellular phase hypoenhancement. In other words, following initial enhancement in the arterial phase (AP, sometimes referred to as "washin"), "washout" is the relative hypointensity of the observation compared with background liver tissue. With extracellular contrast media (ECCM), this hypoenhancement can be assessed either in the portal venous phase (PVP) or the delayed phase (sometimes referred to as the "equilibrium" phase and obtained at about $3 \mathrm{~min}$ ) or both. For gadoxetic acid, the hypoenhancement must be assessed only in the PVP; hypointensity in the transitional phase (TP) of gadoxetic acid-equivalent in post-injection time to the delayed phase of ECCM-does not qualify as washout. The theoretical rationale for this is that lesion hypointensity in the TP could be due to one (or both) of two things: true washout, or simply the increasing enhancement of the surrounding liver from intrahepatocellular contrast uptake [10-13] (Fig. 1).

In contrast to LI-RADS v2018, which restricts the interpretation of washout to the PVP only in order to maintain high specificity [9], the Korean Liver Cancer Study Group (KLCSG-NCC) guidelines v2014 allow identification of washout in the PVP or the TP [14], and the Liver Cancer Study Group of Japan (LCSGJ) v2014 permits identification of washout in the PVP, TP, and even in the hepatobiliary phase (HBP) of gadoxetic acid [15]. The approach advocated by these Asian guidelines increases the sensitivity but decreases the specificity of the diagnosis of HCC (see below). It can also lead to incorrect interpretation of so-called pseudo-washout in non-hepatocytecontaining lesions (e.g. high-flow haemangiomas and hypervascular cholangiocarcinomas).

Four retrospective studies from South Korea assessed the specificity and sensitivity of lesion washout during the PVP alone (defined as 50-60 s after gadoxetic acid injection) or in combination with hypointensity in the TP (3 min after gadoxetic acid injection) for diagnosing HCC [16-19]. Washout in the PVP provided high specificity for diagnosis of HCC in patients with chronic liver disease, while washout in the PVP combined with hypointensity in the TP lowered the specificity (from 97.9 to $86.3 \%$ [18]), 92.9 to $78.6 \%$ [19], and 100 to $94.9 \%$ [17]). In contrast, the sensitivity for the detection of HCC increased when washout in the PVP was combined with hypointensity in the TP (from 70.9 to $86.6 \%, 66.7$ to $72.9 \%$, and 63.6 to $76.6 \%$, respectively). At the time of the Liver Forum, no published data were identified on alternative timings of hypointensity assessment in the TP, e.g. 2 min after injection, and no study had been reported on the changes in specificity and sensitivity of diagnosis in the context of LI-RADS criteria, i.e. after excluding LR-1 
Fig. 1 Hypoenhancement of tumour tissue relative to liver: defined as washout (WO) in the portal venous phase (PVP) or delayed phase (DP) with ECCM, but as washout in the PVP alone - not the transitional phase (TP) — with gadoxetic acid. AP, arterial phase; ECCM, extracellular contrast medium
Time after injection

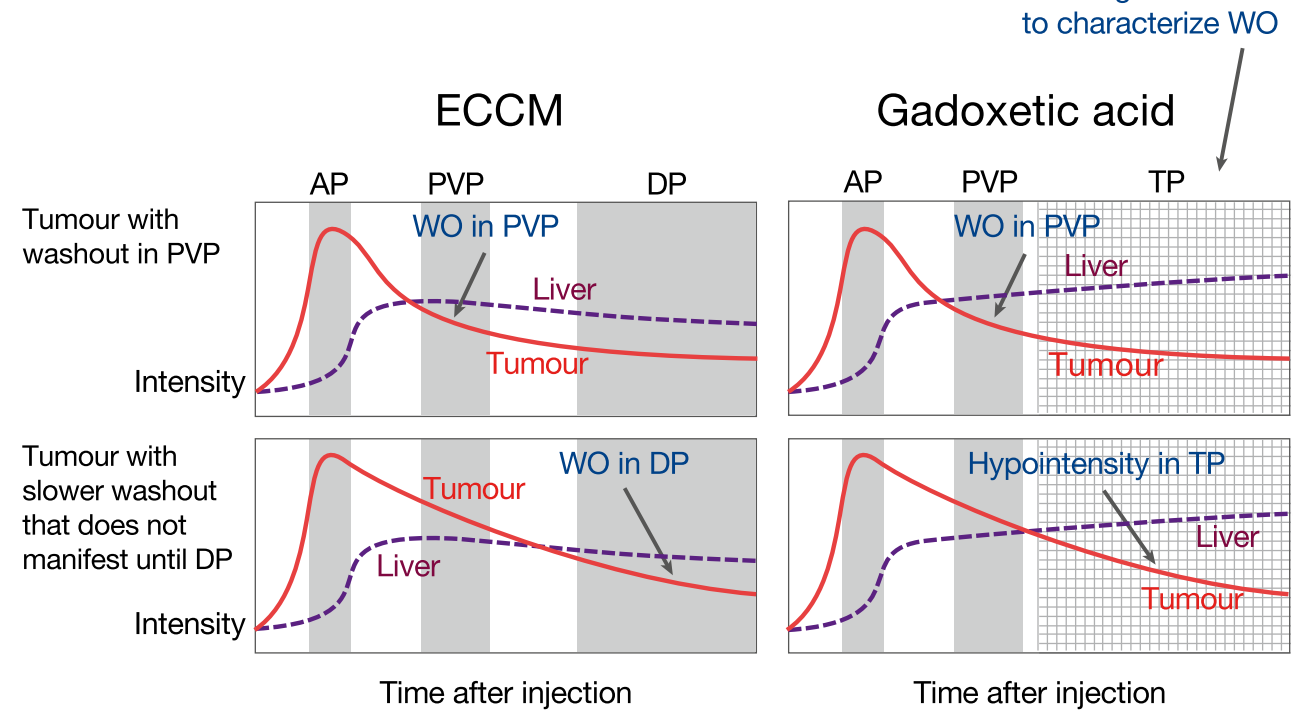

TP images not used to characterize WO
Time after injection (definitely benign), LR-2 (probably benign), and LR-M (probably or definitely malignant but not HCC-specific).

Subsequently published studies have emphasised the increased sensitivity with limited loss of specificity if hypointensity on the HBP is included for diagnosing HCC [20-23].

Consensus statement 1 To maximise specificity for HCC diagnosis using gadoxetic acid-enhanced MRI in patients with cirrhosis or other risk factors and based on the current literature, "washout" of gadoxetic acid should be assessed only in the PVP and not in the 3-min TP. [72/78 (92.3\%) agreement]

Consensus statement 2 Further research is needed to understand the TP using gadoxetic acid. In particular, research is needed to define the physiological beginning and end of the TP based on imaging features rather than fixed time points. Relevant imaging features are likely to include the relative signal intensity of liver parenchyma in comparison to intrahepatic vessels. [81/88 (92.1\%) agreement]

Consensus statement 3 Further research is needed to define when post-AP hypointensity can no longer be interpreted as "washout" with gadoxetic acid-enhanced MRI. Because the temporal signal intensity of gadoxetic acid-enhanced MRI varies across individuals, imaging features (as informed by Consensus statement 2) should be used in this definition, if possible, instead of fixed time points. [81/88 (92.1\%) agreement]

\section{Specificity of the hepatobiliary phase to diagnose HCC}

Kim et al [24] reported that the detection sensitivity for high-grade dysplastic nodules (HGDN), early HCC, and progressed HCC was significantly higher with gadoxetic acid-enhanced MRI than with multidetector computed tomography (CT). Most HGDNs (82.4\%) and early HCCs (76.2\%) demonstrated hypointensity on HBP images.

Hyperenhancement in the AP and washout are described as the most specific imaging features for the diagnosis of HCC in gadoxetic acid-enhanced MRI [25]. Bartolozzi et al reported that lesion enhancement on vascular dynamic and HBP imaging significantly correlated with the histological diagnosis of HCC $(p<0.0001)$ [25]. Golfieri et al found that HBP hypointensity by itself was the strongest MRI marker of malignancy in atypical cirrhotic nodules in gadoxetic acid-enhanced MRI; HBP hypointensity alone had $88 \%$ sensitivity, $97 \%$ specificity, $91 \%$ negative predictive value, and $93 \%$ diagnostic accuracy, significantly superior to any other MRI feature alone or combined [26]. Yoon et al described in a retrospective study of gadoxetic acid-enhanced MRI that a significant proportion of nonhypervascular HBP hypointense nodules $(\geq 1 \mathrm{~cm}$ in diameter) in patients with cirrhosis showed malignant features on pathology $(73.1 \%)$ [27]. In another group of patients whose hypointense HBP nodules were followed up for at least 12 months (mean $19 \pm 10$ months), 32.7\% of nodules developed hypervascularity and $78.8 \%$ showed at least one imaging feature considered to indicate malignant change [27]. However, hypointensity in the HBP by itself cannot differentiate HGDN from early or progressed HCC.

Recommendations on terminology for nodules without AP hyperenhancement and with HBP hypointensity in chronic liver disease have subsequently been published by Motosugi et al on behalf of the LI-RADS HBA Working Group [28].

Consensus statement 4 Hypointensity in the HBP of gadoxetic acid-enhanced MRI is the most sensitive 
imaging feature for the diagnosis of an HGDN, an early HCC, or a progressed HCC in a patient at risk for HCC. Notes: (1) This statement applies regardless of vascularity in the AP; (2) hypointensity by itself has other potential diagnoses (e.g. haemangiomas), besides HCC. [74/89 (83.2\%) agreement]

Consensus statement 5 Using multiparametric MRI (including T2-weighted [T2W] and diffusion-weighted imaging [DWI]), high specificity ( $>90 \%$ ) for the diagnosis of HCC can be achieved when hypointensity in the HBP is associated with solid arterial enhancement, regardless of venous washout, in patients with liver cirrhosis. Note: A small percentage of such lesions may represent other entities, e.g. cholangiocarcinoma or combined HCC-cholangiocarcinoma, while cysts and haemangiomas can be ruled out by the signal in T2W and DWI sequences. [74/87 (85.1\%) agreement]

Consensus statement 6 As a significant number of hypovascular nodules $(>1 \mathrm{~cm})$ that are hypointense on HBP are, or eventually become (by 6-12 months), HCC in patients with liver cirrhosis, close observation or intervention is recommended. Note: The current statement reflects practice differences around the world, including the availability of surgery, ablation, and biopsy. [80/88 (90.9\%) agreement]

\section{Detection of venous invasion with gadoxetic acid-enhanced MRI}

Macrovascular invasion is a characteristic feature of advanced $\mathrm{HCC}$, indicating high risk for metastasis, recurrence, liver functional impairment, and poor prognosis (Barcelona Clinic Liver Cancer [BCLC] stage C) [29]. Microvascular invasion is increasingly recognised as a risk factor for intrahepatic metastases in BCLC stage B/A HCC patients, and even stage 0 HCC patients [30, 31].

Certain imaging features on gadoxetic acid-enhanced MRI in the HBP have been shown in retrospective studies to be predictive for microvascular invasion with high specificity, including the presence and degree of peritumoural hypointensity [13, 32, 33], peritumouraldecreased uptake area [33], and an irregular and nonsmooth tumour margin [34]. The presence of intratumoural fat correlated negatively with microvascular invasion, suggesting intratumoural fat may indicate a lower risk for microvascular invasion [35]. However, these findings need confirmation in independent studies.

Consensus statement 7 In $\mathrm{HCC}$ patients, retrospective single-centre studies suggest that peritumoural HBP hypointensity may predict the presence of microvascular invasion. Prospective multicentre studies are needed to validate peritumoural HBP phase hypointensity for predicting microvascular invasion in $\mathrm{HCC}$, especially in those with $\mathrm{HCC}<2 \mathrm{~cm}$. [39/43 (90.7\%) agreement]

\section{Differential diagnosis of HCC}

The key imaging findings of HCC $(1-2 \mathrm{~cm})$ in LI-RADS v2018 are arterial phase hyperenhancement (APHE, washin), PVP or delayed phase washout, capsular appearance, and threshold growth [8]. These criteria are based on dynamic CT or on MRI with either ECCM (PVP or delayed washout) or gadoxetic acid (PVP washout only) [8]. ECCM and gadoxetic acid are both gadolinium-based contrast agents, but they are not interchangeable because of the differences in pharmacokinetics, dosage, and mechanism of action [36].

Two meta-analyses showed superior sensitivity and positive predictive value (PPV) or negative likelihood ratio for gadoxetic acid-enhanced MRI compared with CT for HCC detection [37, 38]. One of the meta-analyses reported equivalent sensitivity of gadoxetic acid and ECCM for HCC detection [38]. However, gadoxetic acid-enhanced MRI, compared with CT and ECCM-based MRI, may provide alternative imaging features that help differentiate classic HCC from non-HCC malignant lesions. One of the most important features of gadoxetic acid-enhanced MRI is the targetoid HBP pattern-i.e. central hyperintensity with peripheral hypointensity in the rim in the HBP - which can assist to differentiate non-HCC malignant lesions (combined HCC-cholangiocarcinoma, intrahepatic cholangiocarcinoma) from $\mathrm{HCC}$; see Consensus statement 9 [8, 39-41].

Consensus statement 8 There is enough evidence (level 2) to recommend gadoxetic acid-enhanced MRI over contrastenhanced CT as the primary imaging modality for imaging HCC worldwide. There is not enough evidence currently to support a similar statement comparing gadoxetic acidenhanced MRI against MRI using ECCM. [60/72 (83.3\%) agreement]

Consensus statement 9 Compared with CT or ECCM-based MRI, gadoxetic acid-enhanced MRI may provide alternative imaging features that help in differentiating classic HCC from non-HCC malignant lesions (combined HCCcholangiocarcinoma, intrahepatic cholangiocarcinoma). One of the most important such features is the targetoid HBP pattern, which increases the likelihood of a nonHCC malignancy and by itself suffices for assigning an LR-M category using LI-RADS. Notes: (1) Due to fibrous or desmoplastic components, the targetoid pattern can be seen in some $\mathrm{HCC}$ and rare benign lesions, e.g. sclerosed haemangiomas; (2) combined tumours and small 
cholangiocarcinomas may overlap in imaging appearance and can be difficult to differentiate from HCC. [66/83 (79.5\%) agreement]

Consensus statement 10 Comparative studies have demonstrated that gadoxetic acid-enhanced MRI provides higher sensitivity with similar specificity for detecting malignancy when compared with contrast-enhanced CT in cirrhotic liver. Prospective studies are needed to compare the diagnostic performance of gadoxetic acid-enhanced MRI with ECCM. [67/ $83(80.7 \%)$ agreement]

\section{Capsule appearance in HCC with gadoxetic acid-enhanced MRI}

An enhancing "capsule" belongs to the major features of HCC in the LI-RADS v2018 guidelines, where it is defined as a peripheral rim of smooth hyperenhancement in the PVP, DP, or TP [8]. The conventional capsule appearance is a useful feature for diagnosing $\mathrm{HCC}$ [42] that has been investigated in a comparative study of gadoxetic acid and gadobenate dimeglumine [43]. However, the capsule appearance may be obscured by the rapid disappearance of contrast medium from the blood pool and by hyperenhancement of the background liver on gadoxetic acid-enhanced MRI, which are associated with inconsistent sensitivities reported to range from around 20 to $90 \%$ [44-47].

A hypointense rim on the HBP phase is considered an ancillary feature in the LI-RADS v2018 guidelines and has the potential to improve detection of the capsule, favouring the diagnosis of HCC in addition to the conventional capsule appearance alone.

Incorporating the assessment of a hypointense rim in the HBP phase significantly improves the sensitivity and accuracy for the detection of a histological capsule compared with conventional capsule assessment (81.5 vs. $57.8 \%$ and 76.1 vs. $59.4 \%$, respectively; $p<0.001$ ) [48]. Combined assessment of the HCC capsule using the conventional technique and the hypointense rim during the HBP can significantly improve the sensitivity and accuracy for the diagnosis of HCC compared with conventional capsule assessment alone $(83.0$ vs. $72.7 \%$ and 84.1 vs. $75.1 \%$, respectively; $p<0.001$ ), with the same specificity $(91.5 \%)$ [48].

Consensus statement 11 The conventional capsule appearance, a major feature in LI-RADS, may be obscured by the rapid disappearance of contrast medium from the blood pool when using ECCM or gadoxetic acid and progressive hyperenhancement of the background liver on gadoxetic acid-enhanced MRI, resulting in inconsistent sensitivities. [73/82 (89.0\%) agreement]
Consensus statement 12 A smooth hypointense rim on the HBP phase, currently considered an ancillary feature in LIRADS, may have the potential to be included as a capsule appearance. [66/83 (79.5\%) agreement]

\section{Detection of HCC foci prior to therapy decisions}

Curative treatment options-including resection, liver transplantation, and locoregional therapy such as radiofrequency ablation (RFA) - are applicable only for early-stage HCC conforming to the Milan Criteria [49, 50]. Precise staging is therefore key to the optimal management of HCC.

In the more recent versions of management guidelines, there has been an increasing role for hepatobiliary contrastenhanced MRI, greater use of follow-up imaging instead of biopsy, and recommendation for a single dynamic study (either CT or MRI) rather than two dynamic imaging modalities for the diagnosis of small-diameter $(<2 \mathrm{~cm}) \mathrm{HCC}[8,51,52]$. For example, in the Asian Pacific Association for the Study of the Liver (APASL) guideline [52], the diagnostic algorithm is based on dynamic patterns and gadoxetic acid-enhanced MRI is included as a first-line diagnostic tool for HCC (Fig. 2).

Geographical differences exist between guidelines, driven largely by differences in treatment practices. In North America and Europe, the greatest concern is for high specificity. Since patients with a diagnosis of HCC may undergo liver transplantation based on imaging criteria alone, stringent diagnostic criteria are used to avoid false-positive HCC diagnoses [8, 29]. In the European Association for Study of Liver-European Organisation for Research and Treatment of Cancer (EASLEORTC) guidelines, the radiological hallmark for HCC is APHE and portal venous/delayed phase washout [29]. In the 2017 American Association for the Study of Liver Diseases (AASLD) guidelines, MRI with ECCM or gadoxetic acid is concluded to provide higher pooled sensitivity and similar specificity compared with CT [51]. However, the AASLD still recommends diagnostic evaluation of HCC with either multiphasic CTor multiphasic MRI because they assume a similar diagnostic performance. While this statement is strongly endorsed by the AASLD guidelines, the supportive evidence level for CT is relatively low. There are no randomised, controlled trials comparing CT, ECCM-enhanced MRI, and gadoxetic acid-enhanced MRI for the diagnosis of HCC in cirrhotic patients.

In contrast to the approach in North America and Europe, in Asia, the primary aim is to maximise the sensitivity of HCC diagnosis. This is justified by the greater use of locoregional ablative therapies in Asia such as percutaneous ethanol injection, RFA, and transarterial chemoembolisation. In the APASL guidelines, gadoxetic acid-enhanced MRI is preferred over ECCMenhanced MRI as a first-line diagnostic test. Hypointensity on HBP imaging can replace washout on ECCM MRI. Typical HCC can be diagnosed by imaging, regardless of its size, by applying the "washin/washout criteria" [52]. 
Fig. 2 Asian Pacific Association for the Study of the Liver (APASL) Guidelines 2017 [52]. From Omata M, et al Hepatol Int. 11:317-370, with permission.

${ }^{\mathrm{a} C}$ Cavernous haemangioma sometimes shows hypointensity on the equilibrium (transitional) phase of dynamic Gd-EOBDTPA MRI (pseudo-washout). It should be excluded by further MRI sequences and/or other imaging modalities. ${ }^{\mathrm{b}}$ Cavernous haemangioma usually shows hypointensity on the hepatobiliary phase of Gd-EOB-DTPA MRI. It should be excluded by other MRI sequences and/or other imaging modalities. CT, computed tomography; DN, dysplastic nodule; Gd-EOB-DTPA, gadoxetic acid; HCC, hepatocellular carcinoma; MRI, magnetic resonance imaging; US, ultrasound

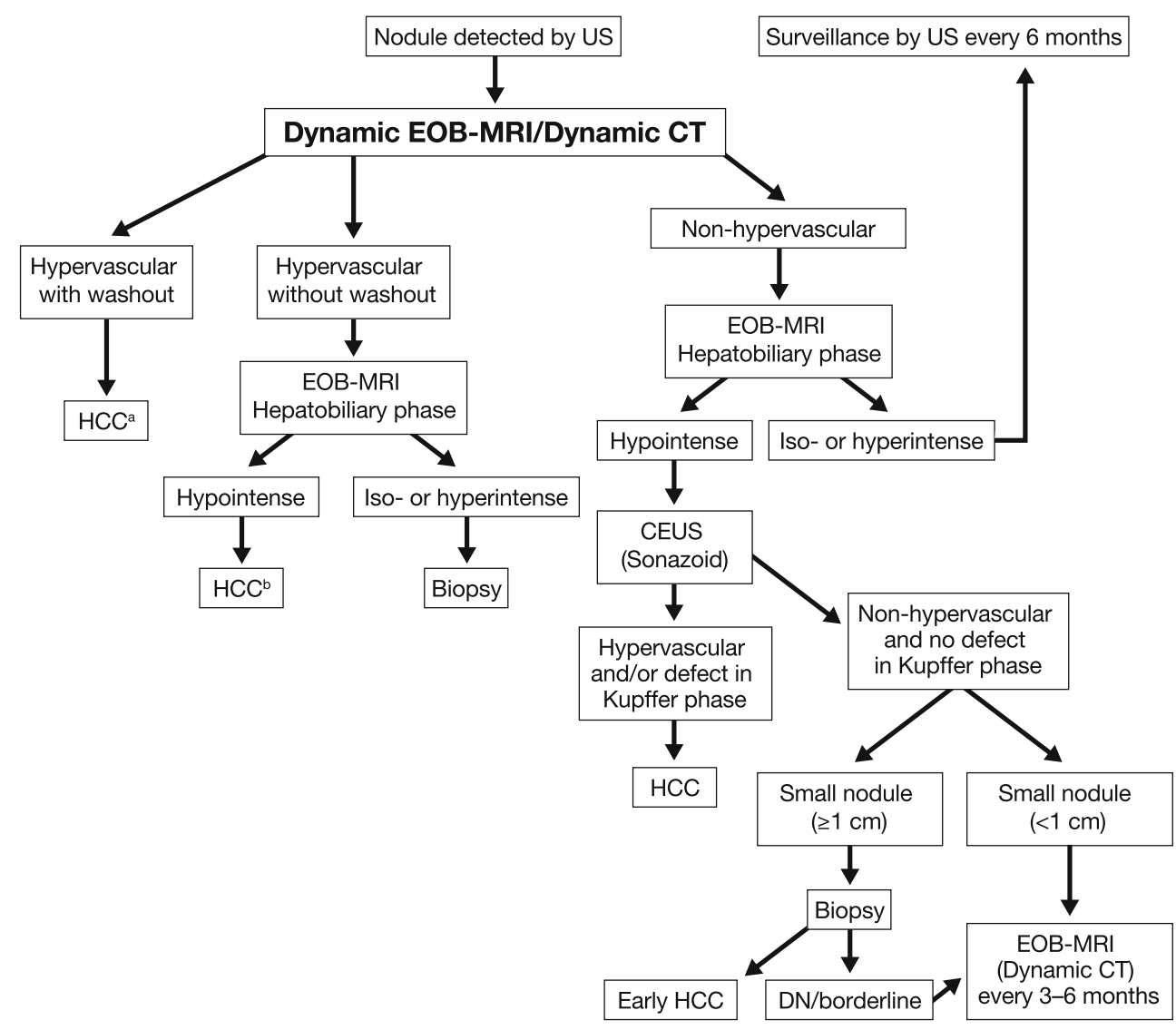

A recent meta-analysis concluded that CT and ECCMbased MRI show similar diagnostic performance for detecting HCC, while gadoxetic acid-enhanced MRI has the highest overall sensitivity and PPV and may be the optimal single method for diagnosis of HCC [37]. Another meta-analysis concluded that MRI with an ECCM or gadoxetic acid has a significantly higher sensitivity ( $82 \%$ vs. $66 \%$ ) and lower negative likelihood ratio $(0.20$ vs. 0.37$)$ versus $C T$, with no differences between the techniques in specificities and positive likelihood ratios [38].

A retrospective comparison against dynamic $\mathrm{CT}$ for staging HCC reported that gadoxetic acid-enhanced MRI provided significantly greater sensitivity $(90.6 \%$ vs. $79.5 \% ; p<0.0001)$ and more accurate BCLC staging $(92.8 \%$ vs. $80.5 \% ; p<0.0001)$. BCLC stage was correctly changed after gadoxetic acidenhanced MRI in $13.8 \%$ patients [53]. Gadoxetic acidenhanced MRI was also superior to $\mathrm{CT}$ for detecting intrahepatic recurrence post-curative surgery in patients with HCC. In a lesion-by-lesion analysis, the sensitivity was significantly higher for gadoxetic acid-enhanced MRI than on multidetector CT ( $p<0.005$, both study reviewers) [54].

Consensus statement 13 Gadoxetic acid-enhanced MRI is an accurate method for the diagnosis and staging of HCC (level 2 evidence in meta-analyses). Although the reported accuracy of gadoxetic acid-enhanced MRI for diagnosis of HCC compares favourably with that of CT or ECCM-enhanced MRI, the quality of the evidence is still insufficient to recommend gadoxetic acidenhanced MRI over CT or ECCM-enhanced MRI in all patient populations. Notes: (1) The statement "in all patient populations" relates to different management patterns in different regions and aims to capture differences in Western versus Asian treatment practices (mentioned above). (2) "Gadoxetic acid-enhanced MRI" refers to the entire package of imaging and not simply to the HBP. [65/87 (74.7\%) agreement]

Consensus statement 14 Gadoxetic acid-enhanced MRI is useful for the preoperative staging of $\mathrm{HCC}$, and also for follow-up after surgery of HCC, as it can detect new lesions with high sensitivity. [82/88 (93.2\%) agreement]

\section{Technical-related issues}

\section{Artefacts in the arterial phase}

Arterial phase (AP) imaging is an essential component in gadoxetic acid-enhanced MRI for making an HCC diagnosis and monitoring treatment response, identifying AP hyperenhancing benign lesions and liver metastases, and assessing hepatic artery anatomy. Artefacts have been described in the literature that may influence image quality in the AP. 
The first description of "acute transient dyspnoea" after administration of gadoxetic acid was published in 2013 [55]. In this single-centre prospective, nonrandomised observational study, 198 patients underwent MRI of the abdomen ( 99 with gadoxetic acid, 99 with gadobenate dimeglumine). A proportion of patients $-14 \%$ in the gadoxetic acid and $5 \%$ in the gadobenate dimeglumine group $(p=0.05)$ - described a temporary, self-limiting phenomenon lasting for 10-20 s, during which they felt as if they "couldn't catch their breath". Concomitantly, there were cases of image quality being severely degraded by patient respiratory motion during the AP, which were more frequent in the gadoxetic acid than in the gadobenate dimeglumine group, both for all patients (17 vs. $2 \%, p=0.0007)$ and for the cirrhotic subpopulation (19 vs. $3 \%, p=0.02)$. This effect did not extend to PVP, transitional phase, or HBP.

A follow-up retrospective study by the same group [56] on 180 patients who underwent gadoxetic acid- and gadobenate dimeglumine-enhanced MRI at different times reported a higher incidence of respiratory motion-related artefact in the AP (transient severe motion [TSM] artefact) associated with gadoxetic acid than gadobenate $(39 \%$ vs. $10 \%, p<0.0001$; severe in $18 \%$ vs. $2 \%$, respectively, $p<0.0001$ ).

In a recent review of the published literature, the prevalence of TSM associated with gadoxetic acid-enhanced MRI of the liver ranged from 5 to $22 \%$ [57]. There are major geographical differences in the prevalence of TSM, with higher rates in patients in the USA compared with those in Asia [58].

The mechanism underlying TSM is unclear. Reported risk factors for TSM in gadoxetic acid-enhanced MRI have included male sex, high body mass index, breath-hold failure $[58,59]$, presence of chronic obstructive pulmonary disease [59], higher (off-label) gadoxetic acid injection doses [59], and history of prior TSM [60, 61].

Proposed solutions to TSM are also widely debated, without current consensus [53-69]. In a recent prospective observational study in 250 consecutive patients, the incidence of acute transient dyspnoea after gadoxetic acid administration was reported in less than $1 \%$ and combination with a multiarterial phase technique significantly reduced the incidence of artefacts [62].

Consensus statement 15 Gadoxetic acid-enhanced MRI has been associated in the literature with artefacts during the AP that are suspected to be secondary to TSM, as recently described. TSM has an estimated prevalence of $2-39 \%$ (mean $15 \%)$. The mechanism of TSM is unclear. [71/76 (93.4\%) agreement]

Consensus statement 16 Several possible solutions have been suggested to minimise the artefact of TSM. These include the use of multiple APs, shortening the acquisition, use of Cartesian and non-Cartesian free-breathing acquisition, contrast dilution, and changes in timing methods. Further research and a consensus are needed to determine which method should be proposed to decrease/eliminate this artefact. [87/ 89 (97.8\%) agreement]

\section{Shortened MRI protocols}

A major objective in protocol optimisation is to provide highquality images within as short a time as possible. Discussion of the optimal protocol for gadoxetic acid-enhanced MRI was included in the First Liver Forum Consensus Manuscript 11 years ago [1]. In the "traditional" protocol (Fig. 3), noncontrast sequences are acquired before gadoxetic acid injection, followed by dynamic evaluation. After a wait of $10 \mathrm{~min}$ in patients with normal liver function and $20 \mathrm{~min}$ in patients with liver cirrhosis or otherwise compromised liver function, the HBP images are acquired. The total protocol duration is approximately $35-40 \mathrm{~min}$.

In an alternative "optimised" protocol using gadoxetic acid (Fig. 4), pre-contrast T2W and DWI are moved before the HBP. This can save 5 or even $8 \mathrm{~min}$ in the protocol. The time saved using the optimised protocol can represent a decrease in patient discomfort and costs relative to the traditional protocol or provide an opportunity to perform optional additional acquisitions.

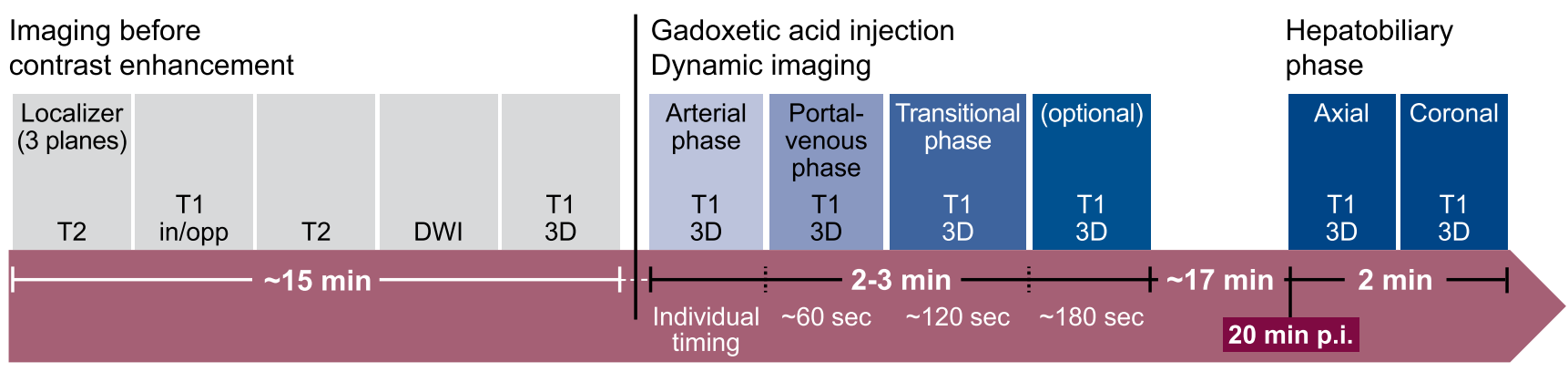

Study duration: $\sim 35-40 \mathrm{~min}$

Fig. 3 Traditional MRI protocol using gadoxetic acid-T1W, T2W, and DWI are performed before gadoxetic acid administration. DWI, diffusionweighted imaging 
a Optimized workflow in cirrhotics

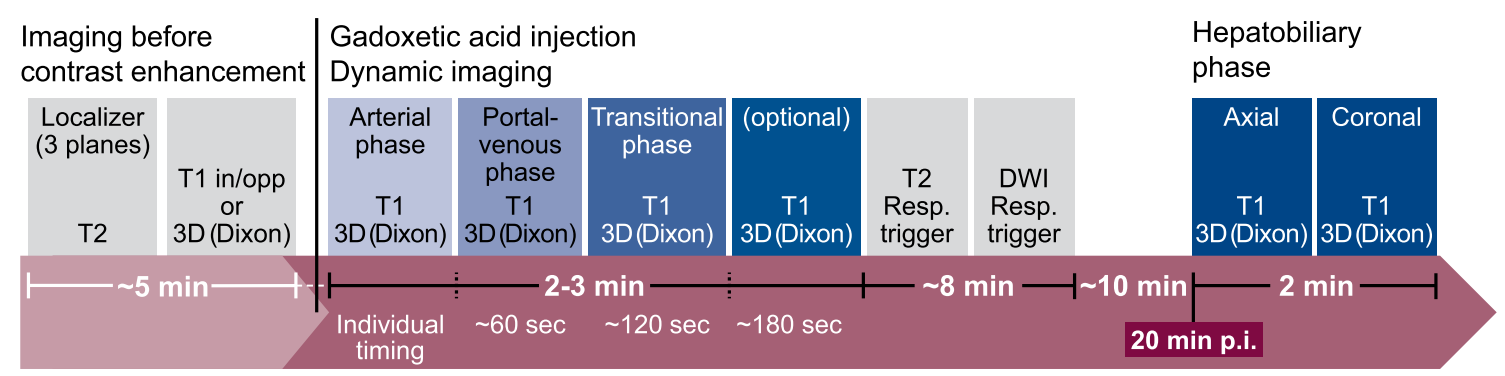

\section{Study duration: $<30 \mathrm{~min}$}

\section{b}

\section{Optimized workflow in non-cirrhotics}

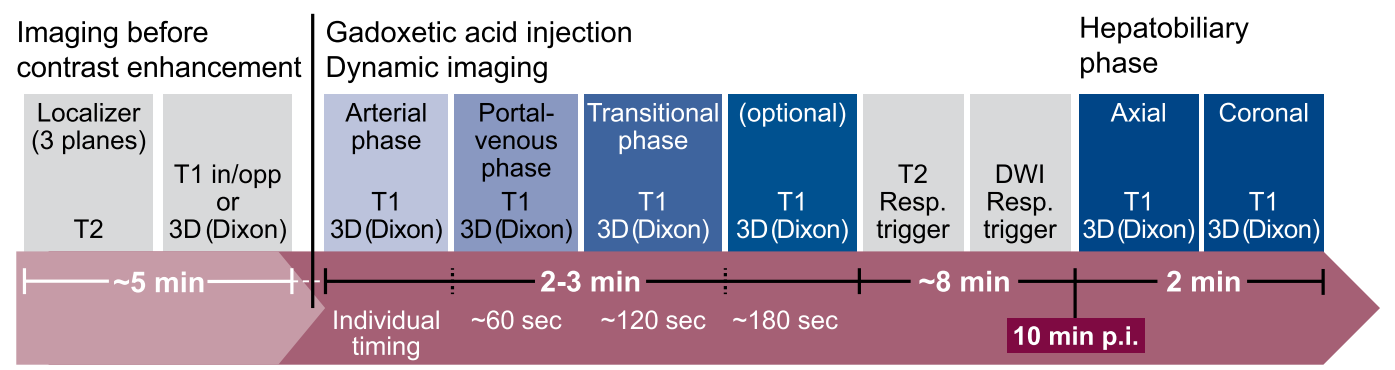

\section{Study duration: $\sim 20 \mathrm{~min}$}

Fig. 4 Optimised MRI protocol using gadoxetic acid. DWI, diffusion-weighted imaging

Studies have demonstrated that if $\mathrm{T} 2 \mathrm{~W}$ and $\mathrm{DW}$ images are acquired after gadoxetic acid injection in the optimised protocol, the same results and comparable diagnostic capability are obtained as in the "traditional" protocol $[63,64]$. An exception to the recommendation to use the optimised protocol is suspected haemangioma, where it is suggested to perform T2 and DWI before gadoxetic acid injection due to the high specificity of T2W MRI in this situation $[65,66]$. The results can inform whether there is subsequently a need to inject gadoxetic acid. Moreover, a T2W-based MR cholangiography cannot be performed after gadoxetic acid injection, unless it is acquired within $5 \mathrm{~min}$ after gadoxetic acid injection.

Additional steps to consider when using an optimised MRI protocol include (1) using bolus timing techniques for optimal enhancement during the dynamic phase [67] and (2) starting the HBP at about 10 min post-injection to save examination time. Although a 20-min delay can improve the signal intensity and liver-to-lesion contrast with benefit in some situations, about a 10-min delay is sufficient in most patients without chronic liver disease [68]. (3) High flip angle delayed HBP imaging is a useful adjunct to standard-enhanced MRI of the liver. It allows for better sensitivity in focal liver detection, particularly for small lesions, and this technique increases the conspicuity of the biliary system, which is an additional benefit of delayed imaging [69-71].

Consensus statement 17 The minimum protocol in the cirrhotic/high-risk HCC-patient for screening and presurgical evaluation/staging incudes:

- $\quad$ T1W in-phase and out-of-phase

- Pre-contrast plus dynamic contrast-enhanced evaluation

- $\quad \mathrm{T} 2 \mathrm{~W}$ and DWI acquisition

- HBP at 20 min

- Optional additional sequences can be used for specific clinical situations or according to institutional preference [71/83 (85.5\%) agreement]

Consensus statement 18 The minimum protocol in the noncirrhotic oncological patient (pre- and post-treatment) consists of:

- $\quad$ T1W in-phase and out-of-phase

- Pre- and post-contrast contrast-enhanced dynamic evaluation 
- $\quad$ T2W and DWI acquisition

- HBP at 10-15 min

- Optional additional sequences can be used for specific clinical situations or according to institutional preference

- Notes: In biliary-related diseases, perform T2 (and DWI) and MR cholangiography before contrast administration. Prospective studies are needed to see if the HBP alone may suffice in the follow-up setting [70/85 (82.4\%) agreement]

An additional protocol suggestion to use gadoxetic acidenhanced MRI when finding an incidental lesion in a healthy patient did not reach overall consensus ( $>50 \%$ agreement) and is not included among the consensus statements.

\section{New sequences for dynamic imaging-current clinical value}

There are current limitations to dynamic contrast-enhanced MRI of liver lesions, including (1) the requirement for an AP and PVP that show an arterial phase-enhancing lesion and washout compatible with HCC and (2) the need for spatial resolution sufficient to detect $1-2-\mathrm{cm}$ tumours combined with temporal resolution sufficient to visualise contrast dynamics within a single breath hold. Additional factors when using gadoxetic acid are an injection rate $1 \mathrm{cc} / \mathrm{s}$ related to a smaller injection volume and a lower total gadolinium dose (usually $0.025 \mathrm{mmol} \mathrm{Gd} / \mathrm{kg} \mathrm{BW}$ ) compared with other ECCM, no prior timing run, and concerns over respiratory motionrelated artefacts.

Solutions to these limitations are being developed. One solution is faster imaging, during a single breath hold, achieved by (1) parallel imaging, through undersampling of the $\mathrm{K}$-space, and use of receiver coils that provide spatial information to unwrap the image [72]; and (2) view-sharing, utilising keyhole imaging techniques, e.g. TRICKS (time-resolved imaging of contrast kinetics) and TWIST (time-resolved angiography with stochastic trajectories), to provide more frequent sampling of the centre of the K-space compared with the periphery. In one study using gadoxetic acid, Pietryga et al used a high parallel acceleration factor to perform multiple APs in one breath hold. The technique recovered most APs that would otherwise have been compromised by transient motion [73].

A second solution is to use a free-breathing approach. Among multiple potential approaches, one is to acquire data in a STAR VIBE (radial volumetric interpolated breath-hold examination) sequence [74]. Fat-suppressed STAR VIBE is acquired with stack-of-stars $\mathrm{K}$-space sampling, which uses conventional sampling in the slice direction and radial sampling in-plane. To speed up radial acquisition, compressed sensing can be used, a method that exploits the compressibility or sparsity of MRI data to reconstruct under-sampled data. This is the concept underlying the GRASP (golden-angle radial sparse parallel) technique, which combines a goldenangle ordering scheme, compressed-sensing reconstruction, and parallel imaging.

The technique acquires continuous data over approximately $3-5 \mathrm{~min}$ in a radial fashion while the patient breathes normally. All required phases can then be reconstructed retrospectively, so that multiple APs and venous phases can be obtained together. A study using this technique to extract perfusion parameters found - as expected - that total plasma flow was reduced but additionally showed that the hepatocellular uptake rate for gadoxetic acid was lower in cirrhotic compared with that in non-cirrhotic liver [75].

XD-GRASP represents a further development in GRASP imaging, utilising retrospective motion-resolved reconstruction for image acquisition, spanning from end-inspiration to end-expiration, to acquire images. Chandarana et al reported that free-breathing, motion-resolved XD-GRASP reconstructions provide high-quality multiphase images in patients undergoing gadoxetic acid-enhanced liver MRI, superior in image quality to standard GRASP reconstructions [76]. Other techniques that enable rapid robust imaging are being investigated and have the potential to help improve the image quality of contrast-enhanced liver exams. Dedicated clinical studies will be required to demonstrate the value of these novel techniques.

Consensus statement 19 Conventional contrast-enhanced multiphasic MRI remains limited with the need for (1) higher spatial resolution (that more closely resembles CT) in the AP as well as higher temporal resolution to shorten the breath hold and (2) greater robustness to eliminate/minimise motionrelated artefacts. [67/72 (93.1\%) agreement]

Consensus statement 20 Recent advances in MRI that enable fast imaging (advanced parallel imaging, view sharing, compressed sensing) and more robust imaging (non-Cartesian imaging) have the potential to improve multiphasic dynamic liver imaging in patients undergoing gadoxetic acid-enhanced liver MRI. However, more technical developments and definitive clinical studies/trials are needed to demonstrate clinical value. [67/68 (98.5\%) agreement]

\section{Summary}

Gadoxetic acid-enhanced MRI has an important role in the care of patients, offering a unique combination of sensitivity and specificity that is recognised in recent management guidelines, and recommended over all other contrast agents and imaging modalities in the Asian guidelines. Optimisation of the acquisition techniques, timing, and other parameters of the arterial, venous, and hepatobiliary phases is an area of ongoing research to further enhance the utility of gadoxetic acid- 
enhanced MRI, while other research is exploring methods to reduce associated artefacts and to utilise the latest advances in multiphasic dynamic image acquisition.

Acknowledgements Medical writing assistance provided by Bill Wolvey at Parexel was funded by Bayer HealthCare.

Funding This study received funding from Bayer HealthCare.

\section{Compliance with ethical standards}

Guarantor The scientific guarantor of this publication is Christoph J. Zech, MD.

Conflict of interest The authors of this manuscript declare relationships with the following companies:

Christoph J. Zech received honoraria for lectures from Bayer HealthCare

Ahmed Ba-Ssalamah nothing to disclose

Thomas Berg nothing to disclose

Hersh Chandarana (1) research support in form of hardware and software from Siemens; (2) co-inventor of GRASP and XD-GRASP with patent on GRASP technique

Gar-Yang Chau nothing to disclose

Luigi Grazioli nothing to disclose

Myeong-Jin Kim nothing to disclose

Jeong Min Lee nothing to disclose

Elmar M. Merkle nothing to disclose

Takamichi Murakami nothing to disclose

Jens Ricke nothing to disclose

Claude B. Sirlin research grants from Bayer HealthCare and is an institutional representative for a consultation agreement between Bayer HealthCare and his university

Bin Song nothing to disclose

Bachir Taouli research grant, Bayer HealthCare

Kengo Yoshimitsu nothing to disclose

Dow-Mu Koh nothing to disclose

Statistics and biometry No complex statistical methods were necessary for this paper.

Informed consent Written informed consent was not required for this study because this is a review paper.

Ethical approval Institutional Review Board approval was not required because this is a review paper.

Methodology Not applicable because this is a review paper

Open Access This article is distributed under the terms of the Creative Commons Attribution 4.0 International License (http:// creativecommons.org/licenses/by/4.0/), which permits unrestricted use, distribution, and reproduction in any medium, provided you give appropriate credit to the original author(s) and the source, provide a link to the Creative Commons license, and indicate if changes were made.

\section{References}

1. Mallone D, Zech CJ, Ayusa C, Bartolozzi C, Jonas E, Tanimoto A (2008) Magnetic resonance imaging of the liver: consensus statement from the 1st International Primovist User Meeting. Eur Radiol Suppl 18(suppl 4):849-864

2. Tanimoto A, Lee JM, Murakami T, Huppertz A, Kudo M, Grazioli L (2009) Consensus report of the 2nd International Forum for liver MRI. Eur Radiol 19(Suppl 5):S975-S989

3. Grazioli L, Lee J, Malfertheiner P, Zech C, Blomqvist L, Merkle E (2010) Consensus report of the Third International Forum for liver magnetic resonance imaging. Invest Radiol 45:S1-S10

4. Lee JM, Zech CJ, Bolondi L et al (2011) Consensus report of the 4th International Forum for gadolinium-ethoxybenzyldiethylenetriamine pentaacetic acid magnetic resonance imaging. Korean J Radiol 12:403-415

5. Zech CJ, Bartolozzi C, Bioulac-Sage P et al (2013) Consensus report of the Fifth International Forum for liver MRI. AJR Am J Roentgenol 201:97-107

6. Sirlin CB, Hussain HK, Jonas E et al (2014) Consensus report from the 6th International Forum for liver MRI using gadoxetic acid. J Magn Reson Imaging 40:516-529

7. Merkle EM, Zech CJ, Bartolozzi C et al (2016) Consensus report from the 7th International Forum for liver magnetic resonance imaging. Eur Radiol 26:674-682

8. LI-RADS Committee. CT/MRI LI-RADS v2017. https://www.acr. org/Clinical-Resources/Reporting-and-Data-Systems/LI-RADS/ CT-MRI-LI-RADS-v2017. Accessed 22 Jan 2019

9. LI-RADS Committee. CT/MRI LI-RADS v2018. https://www.acr. org/Clinical-Resources/Reporting-and-Data-Systems/LI-RADS/ CT-MRI-LI-RADS-v2018. Accessed 22 Jan 2019

10. Choi JY, Lee JM, Sirlin CB (2014) CT and MR imaging diagnosis and staging of hepatocellular carcinoma: part II. Extracellular agents, hepatobiliary agents, and ancillary imaging features. Radiology 273:30-50

11. Hope TA, Fowler KJ, Sirlin CB et al (2015) Hepatobiliary agents and their role in LI-RADS. Abdom Imaging 40:613-625

12. Kang Y, Lee JM, Kim SH, Han JK, Choi BI (2012) Intrahepatic mass-forming cholangiocarcinoma: enhancement patterns on gadoxetic acid-enhanced MR images. Radiology 264:751-760

13. Kim KA, Kim MJ, Jeon HM et al (2012) Prediction of microvascular invasion of hepatocellular carcinoma: usefulness of peritumoral hypointensity seen on gadoxetate disodium-enhanced hepatobiliary phase images. J Magn Reson Imaging 35:629-634

14. Korean Liver Cancer Study Group (KLCSG), National Cancer Center, Korea (NCC) (2015) 2014 Korean Liver Cancer Study Group-National Cancer Center Korea practice guideline for the management of hepatocellular carcinoma. Korean J Radiol 16: 465-522

15. Kudo M, Matsui O, Izumi N et al (2014) JSH consensus-based clinical practice guidelines for the management of hepatocellular carcinoma: 2014 update by the Liver Cancer Study Group of Japan. Liver Cancer 3:458-468

16. Choi SH, Byun JH, Lim YS et al (2016) Diagnostic criteria for hepatocellular carcinoma $3 \mathrm{~cm}$ with hepatocyte-specific contrastenhanced magnetic resonance imaging. J Hepatol 64:1099-1107

17. Choi SH, Lee SS, Kim SY et al (2017) Intrahepatic cholangiocarcinoma in patients with cirrhosis: differentiation from hepatocellular carcinoma by using gadoxetic acid-enhanced MR imaging and dynamic CT. Radiology 282:771-781

18. Joo I, Lee JM, Lee DH, Jeon JH, Han JK, Choi BI (2015) Noninvasive diagnosis of hepatocellular carcinoma on gadoxetic acid-enhanced MRI: can hypointensity on the hepatobiliary phase be used as an alternative to washout? Eur Radiol 25:2859-2868

19. Kim R, Lee JM, Shin CI et al (2016) Differentiation of intrahepatic mass-forming cholangiocarcinoma from hepatocellular carcinoma on gadoxetic acid-enhanced liver MR imaging. Eur Radiol 26: $1808-1817$

20. Joo I, Lee JM, Lee DH, Jeon JH, Han JK (2019) Retrospective validation of a new diagnostic criterion for hepatocellular 
carcinoma on gadoxetic acid-enhanced MRI: can hypointensity on the hepatobiliary phase be used as an alternative to washout with the aid of ancillary features? Eur Radiol 29:1724-1732

21. Kim DH, Choi SH, Kim SY, Kim MJ, Lee SS, Byun JH (2019) Gadoxetic acid-enhanced MRI of hepatocellular carcinoma: value of washout in transitional and hepatobiliary phases. Radiology 292: 270

22. Renzulli M, Biselli M, Brocchi S et al (2018) New hallmark of hepatocellular carcinoma, early hepatocellular carcinoma and high-grade dysplastic nodules on Gd-EOB-DTPA MRI in patients with cirrhosis: a new diagnostic algorithm. Gut 67:1674-1682

23. Vernuccio F, Cannella R, Meyer M et al (2019) LI-RADS: diagnostic performance of hepatobiliary phase hypointensity and major imaging features of LR-3 and LR-4 lesions measuring 10-19 mm with arterial phase hyperenhancement. AJR Am J Roentgenol. https://doi.org/10.2214/AJR.18.20979

24. Kim BR, Lee JM, Lee DH et al (2017) Diagnostic performance of gadoxetic acid-enhanced liver MR imaging versus multidetector $\mathrm{CT}$ in the detection of dysplastic nodules and early hepatocellular carcinoma. Radiology 285:134-146

25. Bartolozzi C, Battaglia V, Bargellini I et al (2013) Contrastenhanced magnetic resonance imaging of 102 nodules in cirrhosis: correlation with histological findings on explanted livers. Abdom Imaging 38:290-296

26. Golfieri R, Grazioli L, Orlando E et al (2012) Which is the best MRI marker of malignancy for atypical cirrhotic nodules: hypointensity in hepatobiliary phase alone or combined with other features? Classification after Gd-EOB-DTPA administration. J Magn Reson Imaging 36:648-657

27. Yoon JH, Lee JM, Yang HK et al (2014) Non-hypervascular hypointense nodules $\geq 1 \mathrm{~cm}$ on the hepatobiliary phase of gadoxetic acid-enhanced magnetic resonance imaging in cirrhotic livers. Dig Dis 32:678-689

28. Motosugi U, Murakami T, Lee JM et al (2018) Recommendation for terminology: nodules without arterial phase hyperenhancement and with hepatobiliary phase hypointensity in chronic liver disease. J Magn Reson Imaging 48:1169-1171

29. European Association for the Study of the Liver (2018) EASL clinical practice guidelines: management of hepatocellular carcinoma. J Hepatol 69:182-236

30. Rodriguez-Peralvarez M, Luong TV, Andreana L, Meyer T, Dhillon AP, Burroughs AK (2013) A systematic review of microvascular invasion in hepatocellular carcinoma: diagnostic and prognostic variability. Ann Surg Oncol 20:325-339

31. Ahn SY, Lee JM, Joo I et al (2015) Prediction of microvascular invasion of hepatocellular carcinoma using gadoxetic acidenhanced MR and (18)F-FDG PET/CT. Abdom Imaging 40:843851

32. Nishie A, Asayama Y, Ishigami K et al (2014) Clinicopathological significance of the peritumoral decreased uptake area of gadolinium ethoxybenzyl diethylenetriamine pentaacetic acid in hepatocellular carcinoma. J Gastroenterol Hepatol 29:561-567

33. Shin SK, Kim YS, Shim YS et al (2017) Peritumoral decreased uptake area of gadoxetic acid enhanced magnetic resonance imaging and tumor recurrence after surgical resection in hepatocellular carcinoma: a STROBE-compliant article. Medicine (Baltimore) 96: e7761

34. Ariizumi S, Kitagawa K, Kotera Y et al (2011) A non-smooth tumor margin in the hepatobiliary phase of gadoxetic acid disodium (GdEOB-DTPA)-enhanced magnetic resonance imaging predicts microscopic portal vein invasion, intrahepatic metastasis, and early recurrence after hepatectomy in patients with hepatocellular carcinoma. J Hepatobiliary Pancreat Sci 18:575-585

35. Min JH, Kim YK, Lim S, Jeong WK, Choi D, Lee WJ (2015) Prediction of microvascular invasion of hepatocellular carcinomas with gadoxetic acid-enhanced MR imaging: impact of intra-tumoral fat detected on chemical-shift images. Eur J Radiol 84:1036-1043

36. Frydrychowicz A, Lubner MG, Brown JJ et al (2012) Hepatobiliary MR imaging with gadolinium-based contrast agents. J Magn Reson Imaging 35:492-511

37. Hanna RF, Miloushev VZ, Tang A et al (2016) Comparative 13year meta-analysis of the sensitivity and positive predictive value of ultrasound, CT, and MRI for detecting hepatocellular carcinoma. Abdom Radiol (NY) 41:71-90

38. Roberts LR, Sirlin CB, Zaiem F et al (2018) Imaging for the diagnosis of hepatocellular carcinoma: a systematic review and metaanalysis. Hepatology 67:401-421

39. Chong YS, Kim YK, Lee MW et al (2012) Differentiating massforming intrahepatic cholangiocarcinoma from atypical hepatocellular carcinoma using gadoxetic acid-enhanced MRI. Clin Radiol 67:766-773

40. Hwang J, Kim YK, Park MJ et al (2012) Differentiating combined hepatocellular and cholangiocarcinoma from mass-forming intrahepatic cholangiocarcinoma using gadoxetic acid-enhanced MRI. J Magn Reson Imaging 36:881-889

41. Park HJ, Kim YK, Park MJ, Lee WJ (2013) Small intrahepatic mass-forming cholangiocarcinoma: target sign on diffusionweighted imaging for differentiation from hepatocellular carcinoma. Abdom Imaging 38:793-801

42. Ishigami K, Yoshimitsu K, Nishihara Y et al (2009) Hepatocellular carcinoma with a pseudocapsule on gadolinium-enhanced MR images: correlation with histopathologic findings. Radiology 250: 435-443

43. Dioguardi Burgio M, Picone D, Cabibbo G, Midiri M, Lagalla R, Brancatelli G (2016) MR-imaging features of hepatocellular carcinoma capsule appearance in cirrhotic liver: comparison of gadoxetic acid and gadobenate dimeglumine. Abdom Radiol (NY) 41:1546-1554

44. Choi YS, Rhee H, Choi JY et al (2013) Histological characteristics of small hepatocellular carcinomas showing atypical enhancement patterns on gadoxetic acid-enhanced MR imaging. J Magn Reson Imaging 37:1384-1391

45. Hope TA, Aslam R, Weinstein S et al (2017) Change in liver imaging reporting and data system characterization of focal liver lesions using gadoxetate disodium magnetic resonance imaging compared with contrast-enhanced computed tomography. J Comput Assist Tomogr 41:376-381

46. Hwang J, Kim YK, Min JH et al (2017) Capsule, septum, and T2 hyperintense foci for differentiation between large hepatocellular carcinoma $(\geq 5 \mathrm{~cm})$ and intrahepatic cholangiocarcinoma on gadoxetic acid MRI. Eur Radiol 27:4581-4590

47. Joo I, Lee JM, Lee DH, Ahn SJ, Lee ES, Han JK (2017) Liver imaging reporting and data system v2014 categorization of hepatocellular carcinoma on gadoxetic acid-enhanced MRI: comparison with multiphasic multidetector computed tomography. J Magn Reson Imaging 45:731-740

48. An C, Rhee H, Han K et al (2017) Added value of smooth hypointense rim in the hepatobiliary phase of gadoxetic acidenhanced MRI in identifying tumour capsule and diagnosing hepatocellular carcinoma. Eur Radiol 27:2610-2618

49. Mazzaferro V, Regalia E, Doci R et al (1996) Liver transplantation for the treatment of small hepatocellular carcinomas in patients with cirrhosis. N Engl J Med 334:693-699

50. Martin P, DiMartini A, Feng S, Brown R Jr, Fallon M (2014) Evaluation for liver transplantation in adults: 2013 practice guideline by the American Association for the Study of Liver Diseases and the American Society of Transplantation. Hepatology 59:1144 1165

51. Heimbach JK, Kulik LM, Finn RS et al (2018) AASLD guidelines for the treatment of hepatocellular carcinoma. Hepatology 67:358 380 
52. Omata M, Cheng AL, Kokudo N et al (2017) Asia-Pacific clinical practice guidelines on the management of hepatocellular carcinoma: a 2017 update. Hepatol Int 11:317-370

53. Choi SH, Byun JH, Kwon HJ et al (2015) The usefulness of gadoxetic acid-enhanced dynamic magnetic resonance imaging in hepatocellular carcinoma: toward improved staging. Ann Surg Oncol 22:819-825

54. Kim KA, Kim MJ, Choi JY et al (2014) Detection of recurrent hepatocellular carcinoma on post-operative surveillance: comparison of MDCT and gadoxetic acid-enhanced MRI. Abdom Imaging 39:291-299

55. Davenport MS, Viglianti BL, Al-Hawary MM et al (2013) Comparison of acute transient dyspnea after intravenous administration of gadoxetate disodium and gadobenate dimeglumine: effect on arterial phase image quality. Radiology 266:452-461

56. Davenport MS, Caoili EM, Kaza RK, Hussain HK (2014) Matched within-patient cohort study of transient arterial phase respiratory motion-related artifact in MR imaging of the liver: gadoxetate disodium versus gadobenate dimeglumine. Radiology 272:123131

57. Well L, Weinrich JM, Adam G, Bannas P (2018) Transient severe respiratory motion artifacts after application of gadoxetate disodium: what we currently know. Rofo 190:20-30

58. Motosugi U (2015) Gadoxetic acid-induced acute transient dyspnea: the perspective of Japanese radiologists. Magn Reson Med Sci 14:163-164

59. Davenport MS, Bashir MR, Pietryga JA, Weber JT, Khalatbari S, Hussain HK (2014) Dose-toxicity relationship of gadoxetate disodium and transient severe respiratory motion artifact. AJR Am J Roentgenol 203:796-802

60. Bashir MR, Castelli P, Davenport MS et al (2015) Respiratory motion artifact affecting hepatic arterial phase MR imaging with gadoxetate disodium is more common in patients with a prior episode of arterial phase motion associated with gadoxetate disodium. Radiology 274:141-148

61. Kim SY, Park SH, Wu EH et al (2015) Transient respiratory motion artifact during arterial phase MRI with gadoxetate disodium: risk factor analyses. AJR Am J Roentgenol 204:1220-1227

62. Grazioli L, Faletti R, Frittoli B et al (2018) Evaluation of incidence of acute transient dyspnea and related artifacts after administration of gadoxetate disodium: a prospective observational study. Radiol Med 123:910-917

63. Chandarana H, Felker E, Taouli B (2010) Is there an effect of GdEOB-DTPA on hepatic T2 signal intensity and apparent diffusion coefficient? Proc Intl Soc Mag Reson Med 2010; 18: Abstract 557

64. Kim YK, Kwak HS, Kim CS, Han YM (2009) Detection and characterization of focal hepatic tumors: a comparison of T2-weighted MR images before and after the administration of gadoxectic acid. J Magn Reson Imaging 30:437-443

65. Cruite I, Schroeder M, Merkle EM, Sirlin CB (2010) Gadoxetate disodium-enhanced MRI of the liver: part 2, protocol optimization and lesion appearance in the cirrhotic liver. AJR Am J Roentgenol 195:29-41

66. Ringe KI, Husarik DB, Sirlin CB, Merkle EM (2010) Gadoxetate disodium-enhanced MRI of the liver: part 1, protocol optimization and lesion appearance in the noncirrhotic liver. AJR Am J Roentgenol 195:13-28

67. Haradome H, Grazioli L, Tsunoo M et al (2010) Can MR fluoroscopic triggering technique and slow rate injection provide appropriate arterial phase images with reducing artifacts on gadoxetic acid-DTPA (Gd-EOB-DTPA)-enhanced hepatic MR imaging? J Magn Reson Imaging 32:334-340

68. Motosugi U, Ichikawa T, Tominaga L et al (2009) Delay before the hepatocyte phase of Gd-EOB-DTPA-enhanced MR imaging: is it possible to shorten the examination time? Eur Radiol 19:26232629

69. Bashir MR, Merkle EM (2011) Improved liver lesion conspicuity by increasing the flip angle during hepatocyte phase MR imaging. Eur Radiol 21:291-294

70. Cho ES, Yu JS, Park AY, Woo S, Kim JH, Chung JJ (2015) Feasibility of 5-minute delayed transition phase imaging with 30 degrees flip angle in gadoxetic acid-enhanced 3D gradient-echo MRI of liver, compared with 20-minute delayed hepatocyte phase MRI with standard 10 degrees flip angle. AJR Am J Roentgenol 204:69-75

71. Haradome H, Grazioli L, Al Manea K et al (2012) Gadoxetic acid disodium-enhanced hepatocyte phase MRI: can increasing the flip angle improve focal liver lesion detection? J Magn Reson Imaging 35:132-139

72. Sodickson DK, Manning WJ (1997) Simultaneous acquisition of spatial harmonics (SMASH): fast imaging with radiofrequency coil arrays. Magn Reson Med 38:591-603

73. Pietryga JA, Burke LM, Marin D, Jaffe TA, Bashir MR (2014) Respiratory motion artifact affecting hepatic arterial phase imaging with gadoxetate disodium: examination recovery with a multiple arterial phase acquisition. Radiology 271:426-434

74. Chandarana H, Block TK, Rosenkrantz AB et al (2011) Freebreathing radial 3D fat-suppressed $\mathrm{T} 1$-weighted gradient echo sequence: a viable alternative for contrast-enhanced liver imaging in patients unable to suspend respiration. Invest Radiol 46:648-653

75. Chandarana H, Block TK, Ream J et al (2015) Estimating liver perfusion from free-breathing continuously acquired dynamic gadolinium-ethoxybenzyl-diethylenetriamine pentaacetic acidenhanced acquisition with compressed sensing reconstruction. Invest Radiol 50:88-94

76. Chandarana H, Feng L, Ream J et al (2015) Respiratory motionresolved compressed sensing reconstruction of free-breathing radial acquisition for dynamic liver magnetic resonance imaging. Invest Radiol 50:749-756

Publisher's note Springer Nature remains neutral with regard to jurisdictional claims in published maps and institutional affiliations. 


\section{Affiliations}

Christoph J. Zech ${ }^{1}$ - Ahmed Ba-Ssalamah ${ }^{2}$ - Thomas Berg ${ }^{3} \cdot$ Hersh Chandarana ${ }^{4,5} \cdot$ Gar-Yang Chau $^{6} \cdot$ Luigi Grazioli $^{7}$. Myeong-Jin Kim ${ }^{8}$ • Jeong Min Lee ${ }^{9}$ Elmar M. Merkle ${ }^{1}$. Takamichi Murakami ${ }^{10}$ • Jens Ricke ${ }^{11} \cdot$ Claude B. Sirlin $^{12}$. Bin Song ${ }^{13} \cdot$ Bachir Taouli $^{14} \cdot$ Kengo Yoshimitsu ${ }^{15} \cdot$ Dow-Mu Koh $^{16}$

1 Radiology and Nuclear Medicine, University Hospital Basel, 4031 Basel, Switzerland

2 Department of Biomedical Imaging and Image-Guided Therapy, Medical University of Vienna, 1090 Vienna, Austria

3 Section of Hepatology, Clinic for Neurology; Department of Internal Medicine, Neurology and Dermatology, University Hospital Leipzig, 04103 Leipzig, Germany

4 Center for Advanced Imaging Innovation and Research (CAI2R), Department of Radiology, New York University School of Medicine, New York, NY 10016, USA

5 Bernard and Irene Schwartz Center for Biomedical Imaging, Department of Radiology, New York University School of Medicine, New York, NY 10016, USA

6 Division of General Surgery, Department of Surgery, Taipei Veterans General Hospital, National Yang-Ming University, Taipei 112, Taiwan

7 Department of Radiology, Spedali Civili di Brescia, 25123 Brescia, Italy

8 Department of Radiology, Yonsei University College of Medicine, Seoul 120-752, South Korea
9 Department of Radiology, Seoul National University Hospital, Seoul 110-744, South Korea

10 Department of Diagnostic and Interventional Radiology, Kobe University Graduate School of Medicine, Kobe 650-0017, Japan

11 Klinik und Poliklinik für Radiologie, Ludwig-MaximiliansUniversität München, Munich, Germany

12 Liver Imaging Group, University of California San Diego, San Diego, CA 92093-0888, USA

13 Department of Radiology, West China Hospital, Sichuan University, Chengdu 610041, People's Republic of China

14 Department of Diagnostic, Molecular and Interventional Radiology and Translational and Molecular Imaging Institute, Icahn School of Medicine at Mount Sinai, New York, NY 10029-6574, USA

15 Department of Radiology, Fukuoka University Faculty of Medicine, Fukuoka City 801-1011, Japan

16 Department of Radiology, Royal Marsden Hospital and The Institute of Cancer Research, London SM2 5NG, UK 